\title{
Influência da rugosidade na resistência à corrosão por pite em peças torneadas de aço inoxidável superaustenítico
}

\author{
Influence of roughness on the pitting corrosion resistance of turned \\ superaustenitic stainless steel
}

\section{Márcio Tadeu Gravalos \\ Sulzer Brasil S/A \\ E-mail:marcio.gravalos@sulzer.com}

\section{Marcelo Martins}

Sulzer Brasil S/A

E-mail:marcelo.martins@sulzer.com

\section{Anselmo Eduardo Diniz \\ Faculdade de Engenharia Mecânica UNICAMP \\ E-mail:anselmo@fem.unicamp.br}

\author{
Paulo Roberto Mei \\ Faculdade de Engenharia Mecânica \\ UNICAMP \\ E-mail: pmei@fem.unicamp.br
}

\section{Resumo}

A resistência à corrosão por pite tem sido correlacionada com as condições morfológicas da superfície: um acabamento superficial liso contribui para diminuir o potencial de iniciação de pites. Esse estudo teve por objetivo investigar a relação entre a resistência à corrosão por pite e a rugosidade em superfícies usinadas do aço inoxidável superaustenítico ASTM A744 grau CN3MN. As amostras fundidas sofreram um torneamento cilíndrico com diferentes combinações das condições de corte, resultando, assim, em diferentes padrões de rugosidade superficial. As superfícies das amostras, então usinadas, foram caracterizadas quanto à rugosidade e ao endurecimento. Após submetidas a um teste por imersão, que acelerava a corrosão, essas superfícies foram examinadas num esteroscópio, tendo sido, também, determinada a perda de peso devido à corrosão. Observou-se que as amostras exibiram diferentes comportamentos com relação à resistência à corrosão, de acordo com as condições de usinagem aplicadas. Uma correlação entre a resistência à corrosão e a rugosidade superficial ficou evidente, assim como à perda de peso devido à formação de pites. Esse estudo identificou que a corrosão pode ser controlada através da seleção dos parâmetros de usinagem apropriados.

Palavras-chave: Acabamento superficial, resistência à corrosão por pite, aço inoxidável superaustenítico, controle de corrosão, parâmetros de usinagem, torneamento, encruamento superficial.

\begin{abstract}
Pitting corrosion resistance has been correlated to the morphological conditions of the surface: a smooth surface finishing decreases the potential for pitting. This study aimed at investigating the relationship between pitting corrosion resistance and surface roughness in the machined surfaces of superaustenitic stainless steel ASTM A744 grade CN3MN. The samples of the casting steel were cylindrically turned sunder different combinations of cutting conditions, producing different surface roughness patterns. The surfaces of the samples, as machined, were characterized by roughness and hardness. After the application of an accelerated immersion corrosion test, these surfaces were examined in a stereoscope and the weight loss by corrosion was also determined. It was revealed that the samples exhibited different corrosion resistance behaviors, according to the machining conditions applied. A correlation between pitting resistance corrosion and machined surface roughness was evident, and also, the weight
\end{abstract}


loss due to the formation of pits. This study has identified that corrosion can be controlled through the selection of appropriate machining parameters.

Keywords: Surface finishing, pitting corrosion resistance, superaustenitic stainless steel, corrosion control, machining parameters, turning, surface work hardening.

\section{Introdução}

O aço inoxidável superaustenítico, composto por uma estrutura predominantemente austenítica, exibe uma melhor resistência à corrosão por pite, por frestas e sob tensão, quando comparado com os aços inoxidáveis austeníticos convencionais da série 300. A liga fundida ASTM A744 CN3MN (1998) é uma das opções de material utilizado na fabricação dos componentes de bombas que operam em ambientes marítimos, pois oferece elevados valores de resistência à corrosão por pite. A usinagem é um dos mais importantes processos que está envolvido na fabricação dos componentes de bombas e por isso torna-se importante conhecer os efeitos deste processo na resistência à corrosão por pite.

Uma superfície com o acabamento o mais liso possível reduz o potencial para a formação de pites (Grubb et al., 2002; Moayed et al., 2003; Ramanathan, 1998). A rugosidade superficial é um conjunto de irregularidades, isto é, pequenas saliências e reentrâncias que caracterizam o acabamento superficial (Shapa, 2000). Durante a usinagem, a seleção dos parâmetros de corte apropriados é muito importante para o controle da corrosão (Braman et al., 2005; Hassiotis et al., 2006). O torneamento é um dos processos de usinagem básico e mais largamente utilizado dentro dos processos de usinagem que as indústrias utilizam (Shaw, 2005) e, também, um dos processos mais comuns em pesquisas experimentais em cortes de metal (Trent et al., 2000). No torneamento, o avanço e o raio de ponta têm uma contribuição geométrica à rugosidade superficial (Diniz et al., 2003).

O objetivo desse estudo é o de determinar a relação entre os parâmetros utilizados no processo de torneamento e a resistencia à formação de corrosão por pite, através da análise da influência da rugosidade superficial e da camada endurecida.

\section{Materiais e métodos}

Nesse estudo, foram utilizadas amostras cilíndricas do aço inoxidável superaustenítico tipo CN3MN, com 95 mm de diâmetro e 200 mm de comprimento, fundidas pelo processo estático e solubilizadas a $1170^{\circ} \mathrm{C}$ por 2 horas (Ritoni, 2007). Na Tabela 1, encontramse a composição química e os valores do PREN (Pitting Resistance Equivalent), valores que determinam a resistência à corrosão por pite conforme ASTM A890/890M (1999), calculados através da equação: $P R E N=C r \%+3,3 M o \%$ $+16 N \%$.

As amostras fundidas, com $212 \pm 11$ HV de dureza, foram submetidas às operações de torneamento em desbaste e acabamento em um torno horizontal CNC Mazak QT25, utilizando fluido de corte com $6 \%$ de emulsão em água, em ambundância. Os parâmetros de usinagem utilizados encontram-se na Tabela 2. Para operação de acabamento, os experimentos foram conduzidos variando-se três parâmetros de usinagem em dois níveis, na seguinte seqüência: o avanço (f), a profundidade de corte $\left(a_{p}\right)$ e o ângulo ortogonal de saída do inserto $\left(\gamma_{0}\right)$. Para a operação de desbaste, variouse o ângulo ortogonal de saída. Os parâmetros velocidade de corte $\left(\mathrm{V}_{\mathrm{c}}\right)$ e raio de ponta da ferramenta $\left(R_{\varepsilon}\right)$ foram fixados (Gravalos et al., 2006). Foram utilizados insertos intercambiáveis de metal duro com tripla cobertura composta de $\mathrm{Ti}(\mathrm{C}, \mathrm{N})+\mathrm{Al}_{2} \mathrm{O}_{3}+\mathrm{TiN}$, na geometria ISO CNMG 120404/12 e fixados no porta-ferramenta ISO PCLNR $2525 \mathrm{M} 1$, com ângulo de folga $(\alpha)$ de $6^{\circ}$ e ângulo de posição $(\chi r)$ de $95^{\circ}$. Para realização dos ensaios e análises das superficies, após a usinagem as amostras foram seccionadas conforme demonstrado na Figura 1.

A rugosidade, na superfície das amostras, foi medida em 3 diferentes posições angularmente equidistante, através do rugosímetro Perthometer M1Mahr, com valor de cut-off de $0,8 \mathrm{~mm}$ e comprimento de amostragem igual a $5 \mathrm{~mm}$.

A medição das microdurezas superficiais baseou-se na norma ASTM E 384-07 (2007). Esse método estabelece a determinação da microdureza dos materiais através de ensaio de micropenetração feito com um penetrador Knoop ou Vickers. As amostras foram embutidas e as medições foram realizadas no microdurômetro Buehler modelo 1600-6300, aplicando-se uma carga de 50 gf durante 10 segundos. As medidas foram tomadas partindo-se da superfície usinada e em direção ao centro. Após a medição da microdureza, as amostras foram atacadas com água-régia e a microestrutura foi observada e fotografada através do microscópio óptico Zeiss, modelo Axiotech.

Para análise da resistência à formação de pites, as amostras (ilustradas na Figura 1) foram, primeiramente,

Tabela 1 - Valores da composição química (\% em peso) e do PRE ${ }_{N}$.

\begin{tabular}{c|c|c|c|c|c|c|c|c|c|c|c}
\hline Elemento & $\mathbf{S i}$ & $\mathbf{C}$ & $\mathbf{M o}$ & $\mathbf{C r}$ & $\mathbf{S i}$ & $\mathbf{N i}$ & $\mathbf{P}$ & $\mathbf{M n}$ & $\mathbf{C u}$ & $\mathbf{N}$ & PREN \\
\hline Amostra & 0,74 & 0,015 & 6,25 & 20,79 & 0,008 & 24,65 & 0,021 & 0,63 & 0,33 & 0,2 & 44,6 \\
\hline \multirow{2}{*}{ Norma } & 1 Máx. & $\begin{array}{c}0,03 \\
\text { Máx. }\end{array}$ & $\begin{array}{c}6,0 \text { a } \\
7,0\end{array}$ & 20 a 22 & $\begin{array}{c}0,01 \\
\text { Máx. }\end{array}$ & $\begin{array}{c}23,5 \text { a } \\
25,5\end{array}$ & $\begin{array}{c}0,04 \\
\text { Máx. }\end{array}$ & 2 Máx. & $\begin{array}{c}0,75 \\
\text { Máx. }\end{array}$ & $\begin{array}{c}0,18 \text { a } \\
0,26\end{array}$ & $\geq 40$ \\
\hline
\end{tabular}


Márcio Tadeu Gravalos et al.

\begin{tabular}{|c|c|c|c|c|c|c|c|c|c|}
\hline \multicolumn{2}{|c|}{ Inserto } & \multicolumn{3}{|c|}{ Condições de corte } & \multirow{2}{*}{$\begin{array}{c}\text { Rugosidade } \\
\mathrm{Ra}(\mu \mathrm{m})\end{array}$} & \multicolumn{2}{|c|}{ Endurecimento superficial } & \multicolumn{2}{|c|}{ Resistência à corrosão } \\
\hline $\begin{array}{c}\mathrm{R}_{\varepsilon} \\
(\mathrm{mm})\end{array}$ & $\begin{array}{l}Y_{\circ} \\
\left({ }^{\circ}\right)\end{array}$ & $\begin{array}{c}f \\
\text { (mm/rot.) }\end{array}$ & $\begin{array}{c}\mathrm{V}_{\mathrm{c}} \\
(\mathrm{m} / \mathrm{min} .)\end{array}$ & $\begin{array}{c}a_{p} \\
(m m)\end{array}$ & & $\begin{array}{l}\text { Dureza máx. } \\
\qquad\left(\mathrm{HV}_{0,05}\right)\end{array}$ & $\begin{array}{c}\text { Camada } \\
(\mu \mathrm{m})\end{array}$ & $\begin{array}{c}\text { Perda de } \\
\text { massa (\%) }\end{array}$ & $\begin{array}{c}\text { Densidade de } \\
\text { pites } \\
\left(n^{\circ} \text { pites } / \mathrm{mm}^{2}\right)\end{array}$ \\
\hline${ }^{*} 1,2$ & *10 & ${ }^{*} 0,25$ & *60 & *2,5 & 1,22 & 464 & 165 & $19 \times 10^{-3}$ & $194 \times 10^{-3}$ \\
\hline 1,2 & 0 & 0,25 & 60 & 2,5 & 2,28 & 471 & 215 & $29 \times 10^{-3}$ & $215 \times 10^{-3}$ \\
\hline 0,4 & 10 & 0,1 & 60 & 0,2 & 0,54 & 428 & 265 & $4 \times 10^{-3}$ & $10 \times 10^{-3}$ \\
\hline 0,4 & 10 & 0,2 & 60 & 0,2 & 0,76 & 365 & 115 & $13 \times 10^{-3}$ & $25 \times 10^{-3}$ \\
\hline 0,4 & 10 & 0,1 & 60 & 0,4 & 0,98 & 433 & 165 & $7 \times 10^{-3}$ & $79 \times 10^{-3}$ \\
\hline 0,4 & 10 & 0,2 & 60 & 0,4 & 1,16 & 371 & 215 & $31 \times 10^{-3}$ & $203 \times 10^{-3}$ \\
\hline 0,4 & 0 & 0,1 & 60 & 0,4 & 0,89 & 419 & 165 & $4 \times 10^{-3}$ & $27 \times 10^{-3}$ \\
\hline 0,4 & 0 & 0,2 & 60 & 0,4 & 1,04 & 401 & 115 & $15 \times 10^{-3}$ & $77 \times 10^{-3}$ \\
\hline
\end{tabular}

*Parâmetros aplicados na operação prévia de desbaste de todas amostras que foram usinadas em acabamento.

submetidas a um teste que acelerava a corrosão, sendo imersas em uma solução de cloreto de ferro aquecida a $50 \pm 2^{\circ} \mathrm{C}$ por um período de 72 horas (Moayed, 2003; Salina-Bravo, 1994). Em seguida, as superfícies foram observadas através de um microscópio óptico de baixa ampliação, onde foi possível quantificar a densidade dos pites formados por unidade de área. Para conhecer o valor da perda de massa, as amostras foram pesadas em uma balança com precisão de 0,001 gramas, antes e depois do teste de imersão.

\section{Resultados e discussões}

\subsection{Análise de rugosidade}

Através dos resultados da medição da rugosidade Ra, que estão mostrados na Tabela 2, pode-se observar que, na operação de acabamento, o crescimento do avanço (f) aumenta a rugosidade (Ra). Quando a profundidade de corte $\left(\mathrm{a}_{\mathrm{p}}\right)$ aumenta, para o mesmo valor de raio de ponta da ferramenta $\left(\mathrm{R}_{\varepsilon}\right)$, a rugosidade $(\mathrm{Ra})$ também aumenta. A variação

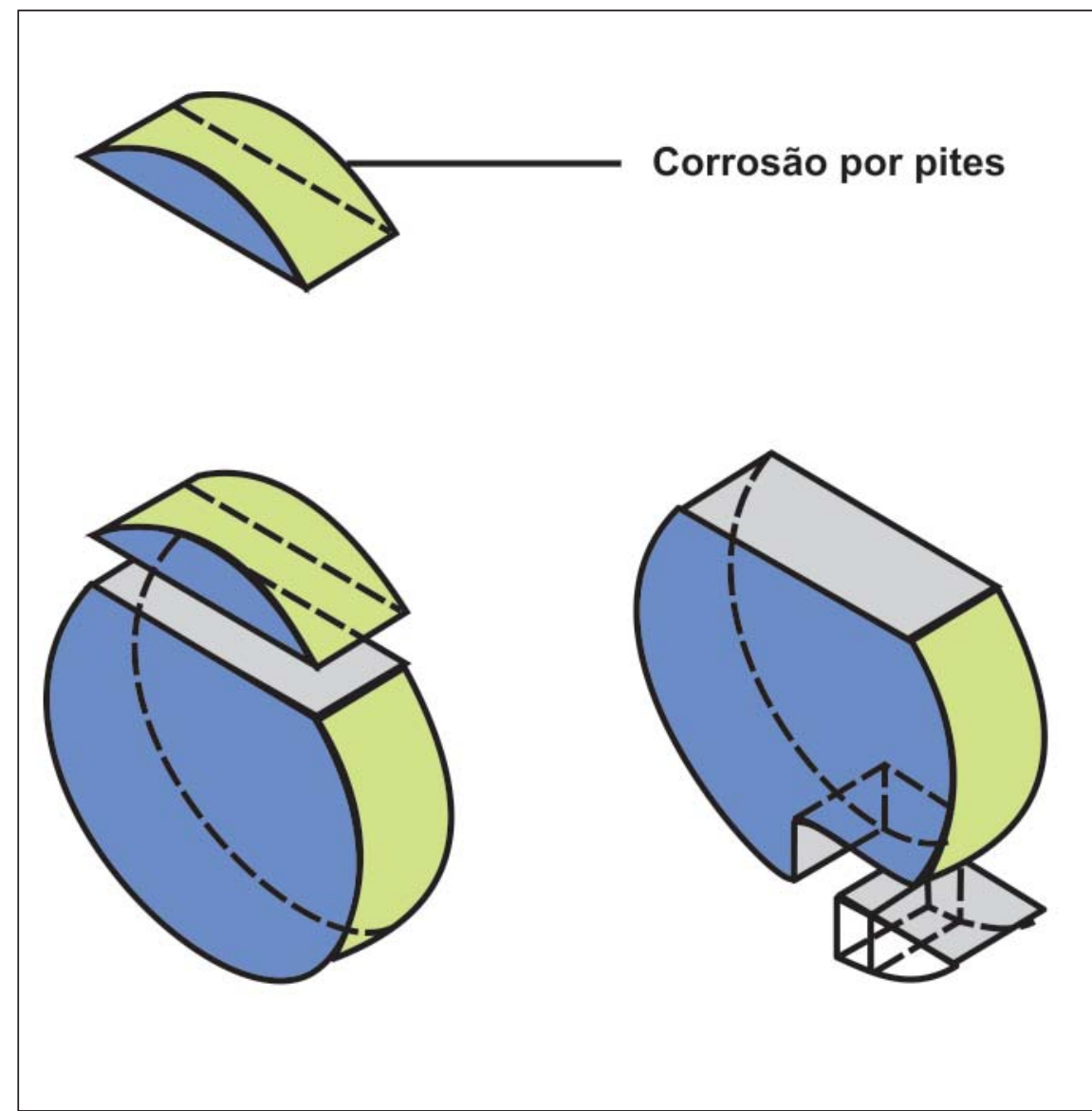

Figura 1 - Amostras seccionadas após a usinagem. 
do ângulo de saída $\left(\gamma_{0}\right)$ teve um efeito pouco significante. $\mathrm{Na}$ operação de desbaste, onde $\mathrm{a}_{\mathrm{p}}$ é bem maior que $\mathrm{R}_{\varepsilon}$, há uma forte influência do $\gamma_{0}$ na rugosidade Ra, que comprova a alta ductilidade desse material usinado. Assim, quando o $\gamma$ diminui, aumenta a taxa de deformação do cavaco e da superfície usinada, o que refletiu no aumento da rugosidade Ra.

\subsection{Análise da dureza superficial}

A Figura 2 é composta da imagem da análise metalográfica e do gráfico do perfil de microdureza de uma das amostras.

Através da Figura 2, pode-se observar o perfil de dureza da camada endurecida formada na superficie usinada em direção ao núcleo. Uma camada superficial de níquel foi aplicada, antes do ensaio de microdureza, para evitar a expansão do material em direção à borda durante a penetração do diamante. As marcas geradas pelo penetrador de dureza Knoop, na estrutura dentrítica, também podem ser observadas na Figura 2.

Os resultados mostrados na Tabela 2 identificam que, em todas as amostras, houve um endurecimento superficial numa camada média de $190 \pm 35 \mu \mathrm{m}$ para o desbaste e de $173 \pm 58$ $\mu \mathrm{m}$ para o acabamento. A dureza máxima alcançada foi, em média, de $468 \pm 5 \mathrm{HV}$, na operação de desbaste, e de $403 \pm 29$ $\mathrm{HV}$, na operação de acabamento. Pode-se notar que a camada superficial endurecida de $0,16 \mathrm{~mm}$, deixada na operação prévia de desbaste foi eliminada na operação posterior de acabamento, pois os valores da profundidade de corte aplicados foram maiores $\left(a_{p}=0,2\right.$ e $\left.0,4 \mathrm{~mm}\right)$ e formou-se uma nova camada endurecida.

\subsection{Análise da resistência à formação de pites}

Os resultados da análise quantitativa dos pites formados na superfície e da perda de massa das amostras durante o teste de corrosão acelerada estão demonstrados na Tabela 2. As imagens da Figura 3 foram obtidas durante a análise superficial em uma das amostras e representam, em diferentes ampliações, a característica dos pites formados isoladamente durante o ensaio de acelaração da corrosão.

Através da Figura 3, pode-se observar um pite típico de forma hemisférica, com diâmetro da ordem de $100 \mu \mathrm{m}$.

\subsection{Correlação entre as respostas obtidas}

Com os resultados da Tabela 2, foi possível construir os gráficos das Figuras 4 e 5, que comparam, respectivamente, a perda de massa e a densidade de pites com a rugosidade Ra.

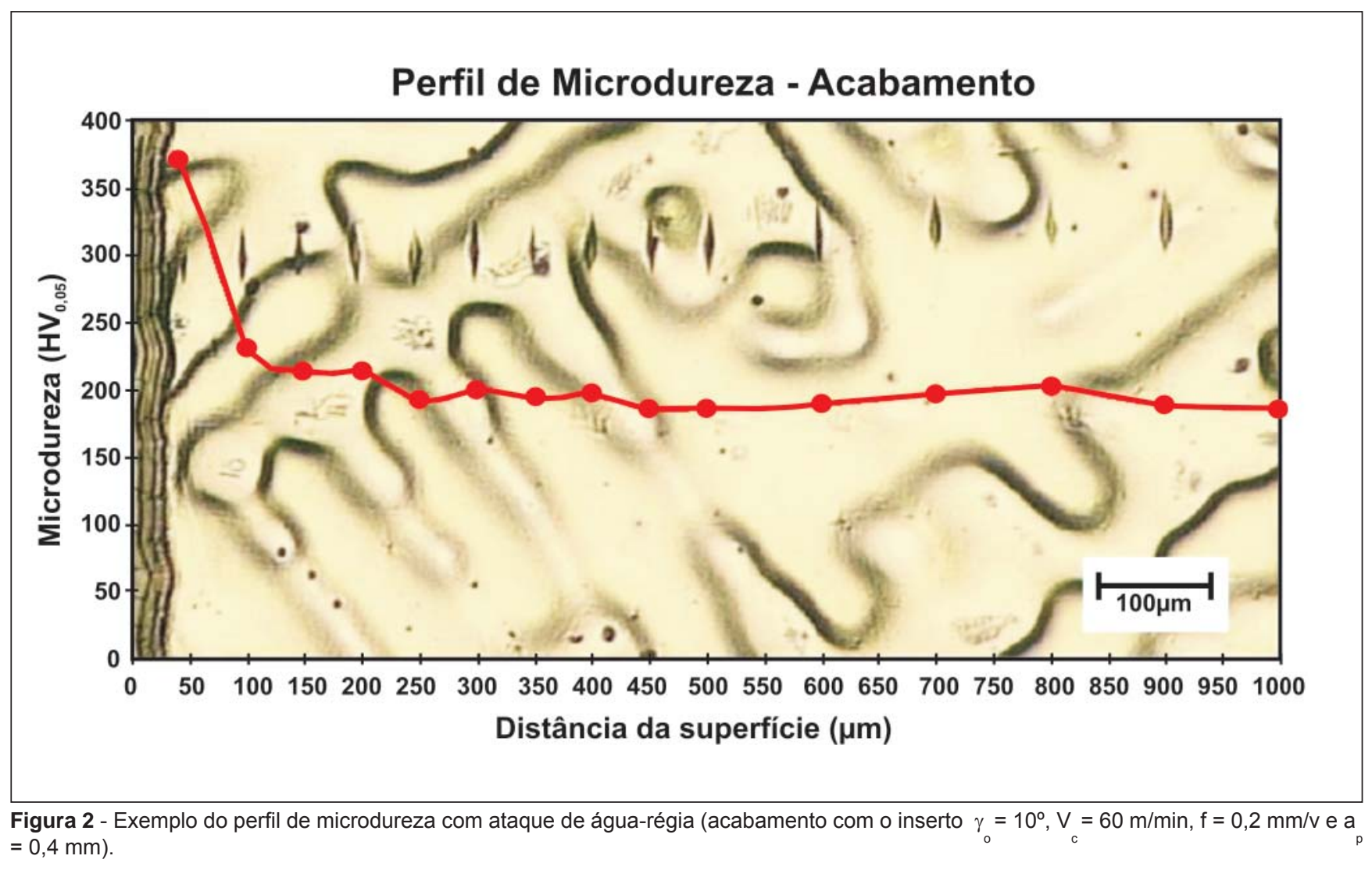




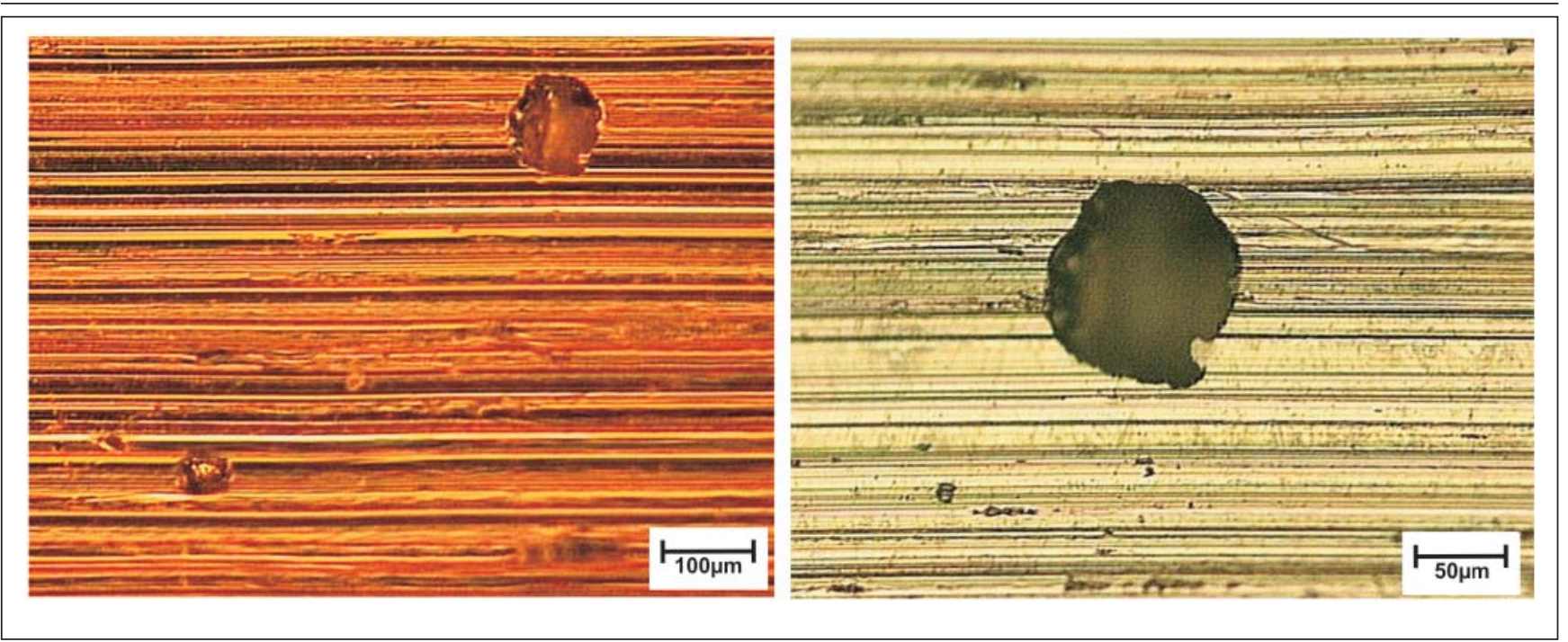

Figura 3 - Pite na superfície com rugosidade $\mathrm{Ra}=2,278 \mu \mathrm{m}$.

Nota-se, nos gráficos da Figuras 4 e 5, que o aumento de perda de massa e da concentração de pites por unidade de área é influenciado pelo aumento da rugosidade Ra. Para o menor valor de rugosidade de $0,54 \mu \mathrm{m}$ (acabamento com $\mathrm{f}=0,1$ $\mathrm{mm} /$ rot. e $\mathrm{a}_{\mathrm{p}}=0,2 \mathrm{~mm}$ ), há pouca perda de massa e de formação de pites. A Figura 6 representa o resultado do efeito do endurecimento superficial na resistência à corrosão por pites.

Observa-se, através da Figura 6, que não é possível identificar uma correlação entre a densidade de pites formados com o tamanho da camada endurecida pela usinagem e nem com a máxima dureza superficial obtida após usinagem.

\section{Conclusões}

1. A formação da uma camada superficial endurecida, o encruamento, no aço inoxidável superaustenítico, ficou evidente em todos os parâmetros de usinagem aplicados durante as operações de torneamento em desbaste e acabamento.

2. O resultado das análises da resistência à formação de pites nas amostras, quando submetidas ao teste de corrosão acelerada, mostraram uma correlação com a rugosidade Ra. Porém não foi possível estabelecer uma correlação com a camada superficial endurecida.

3. Um acabamento superficial liso, ou seja, uma baixa rugosidade Ra, obtida através da seleção dos parâmetros de usinagem apropriados, contribuíram no controle de formação dos pites em superficies usinadas.

\section{Agradecimentos}

Ao Prof. Dr. Luiz Carlos Casteletti e ao Sr. Eliezer Dias Francisco, do Departamento de Engenharia de Materiais da Escola de Engenharia São Carlos - USP, pela colaboração na

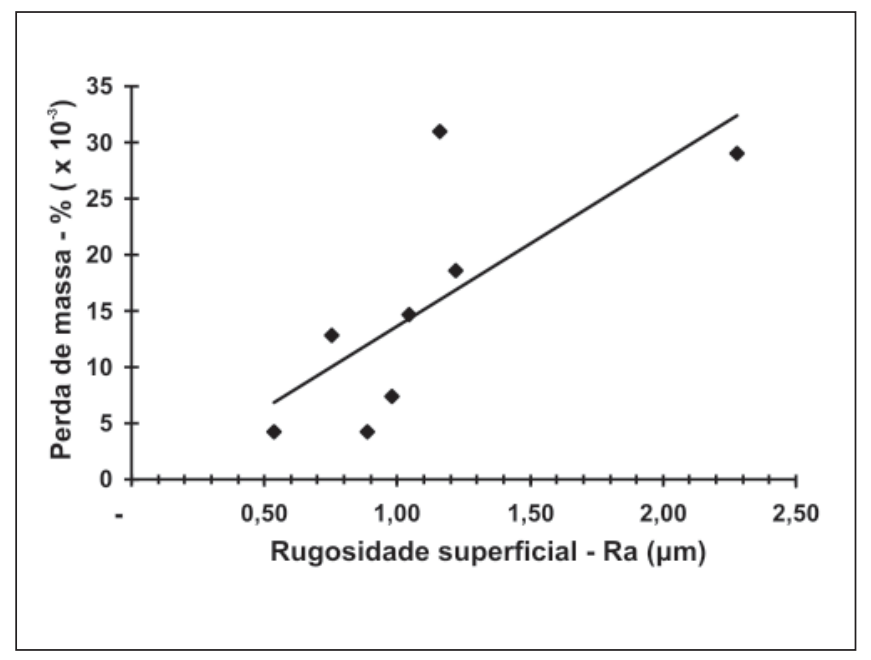

Figura 4 - Perda de massa para diferentes rugosidades Ra.

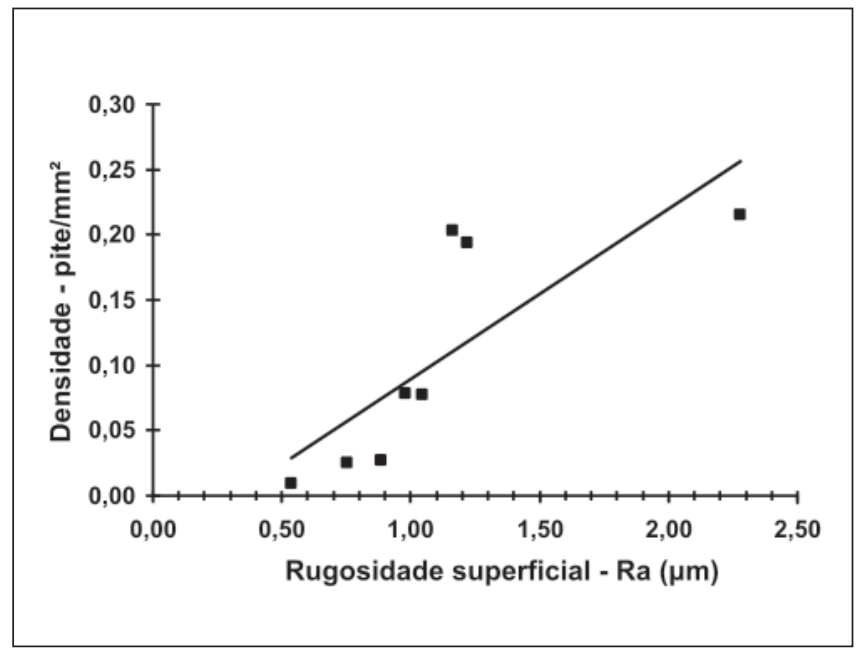

Figura 5 - Densidade de pites para diferentes rugosidades Ra. 
realização dos ensaios de microdureza e também ao Prof. Dr. Lalgudi Venkataraman Ramanathan e ao Sr. Olandir Vercino Correa, do Centro de Ciências e Tecnologia de Materiais - IPEN, pela colaboração na realização dos ensaios de corrosão.

\section{Referências}

\section{bibliográficas}

ASTM A 351/351M-03. Standard Specification for Casting, Austenitic, AusteniticFerritic (Duplex), for Pressure-Containing Parts. 2003, p.137-141.

ASTM A743/743M. Standard specification for casting, iron-chromium, iron-chromium-nickel, corrosion resistant, for general application. U.S.A, 1998. 6p.

ASTM A890/890M - 99. Standard Specification for Castings, Iron-ChromiumNickel-Molybdenum corrosion-resistant, duplex (austenitic/ferritic) for general application, U.S.A., 1999. 4p.

ASTM E 384-07. Standard Test Method for Microindentation Hardness of Materials, U.S.A, 2007. 33p.

BRAMAM, C., BEN RHOUMA, A., LÉDION, J., SIDHOM, H. Effect of machining Conditions on Residual Stress Corrosion Cracking of 316L SS. Materials Science Forum, v. 490-491, p. 305-310. Switzerland, 2005.

DINIZ, A.E., MARCONDES, F.C., COPPINI, N.L. Tecnologia da Usinagem dos Metais. (4.ed.). São Paulo: Editora Artliber, 2003. 248 p.

GRAVALOS, M.T., MARTINS, M., DINIZ, A.E., MEI, P.R. Efeito da usinagem na estrutura e propriedades mecânicas do aço superaustenítico ASTM A351 CN3MN, REM-Revista Escola de Minas, Brasil, p. 83-88, 2006.

GRUBB, J. F., DEEMER, D. E., AL6XN®ALLOY, Allegheny Ludlum, USA, 2002. p. 2-7.

HASSIOTIS, N.D., PETROPOULOS, G.P. Influence of surface roughness on corrosion resistance of turned carbon steel

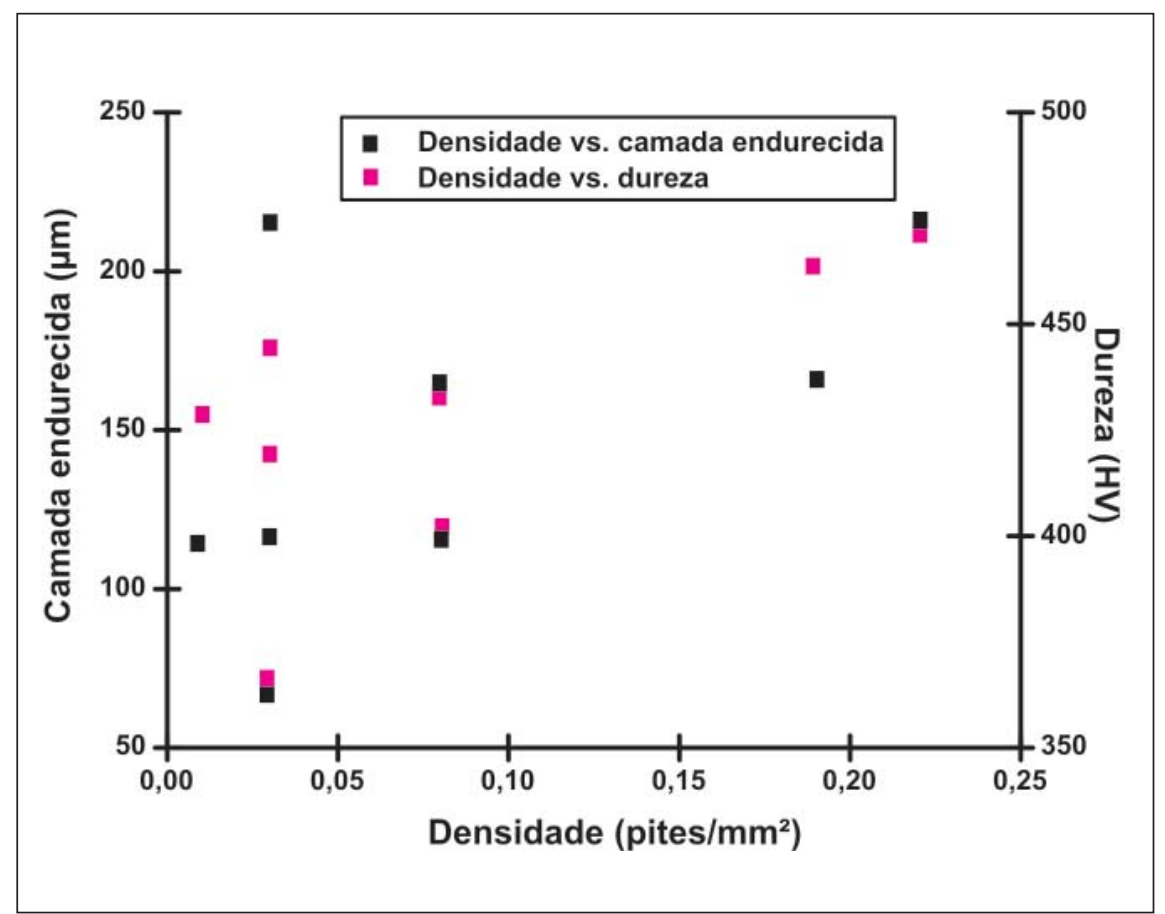

Figura 6 - Relações entre os efeitos da camada endurecida provocado pela usinagem (medidos pela máxima dureza superficial e tamanho de camada endurecida) com a formação de pites.

parts, International Journal of Machining and Machinability of Materials, v. 1, n. 2, p. 202-212, 2006.

MOAYED, M. H., LAYCOCK, N. J., NEWMAN, R. C. Dependence of the Critical Pitting Temperature on surface roughness. Corrosion Science, Elsevier, v. 45, n.6, p. 12031216, 2003.

RAMANATHAN, L. V. Corrosão e seu controle. São Paulo: Hemus Editora Ltda. 339 p. RITONI, M. Efeitos dos tratamentos térmicos na microestrutura e propriedades mecânicas do aço superaustenítico ASTM A 744 Gr. CN3MN. Campinas: Faculdade de Engenharia Mecânica da Universidade de Campinas, 2007. 128 p. (Dissertação de Mestrado).

SALINA-BRAVO, V.M., NEWMAN, R.C. An alternative method to determine critical pitting temperature of stainless steels in ferric chloridhe solution. Corrosion Science, Elsevier, UK, v. 36, n.1, p.66-77, 1994

SHAPA TECHNICAL BULLETIN, The Surface Finish of Stainless Steel on Fabricated Itens, n. 1, p.10, 2000

SHAW, M.C., Metal cutting principles, (2.ed.). New York: Oxford University Press, 2005. $432 \mathrm{p}$.

TECHNICAL DATA OF STAINLESS STEEL AL-6XN@ALLOY, Allegheny Ludlum Corporation, Pittsburgh, PA, USA, 1998. 56 p.

TRENT, E.M., WRIGHT, P. K. Metal cutting.(2.ed.). Butterworth-Heinemann, Woburn, USA, 2000, $446 \mathrm{p}$.

Artigo recebido em 15/06/2009 e aprovado em 19/01/2010.

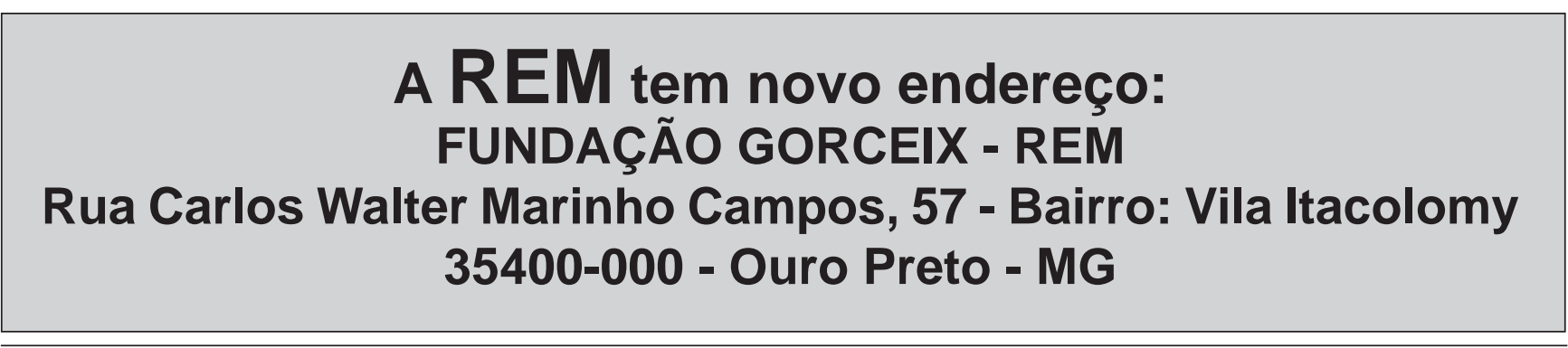

